\title{
CARACTERIZACIÓN MORFOLÓGICA Y BIOQUÍMICA DE CEPAS DE Rhizobium COLECTADAS EN FRIJOL COMÚN SILVESTRE Y DOMESTICADO
}

\author{
MORPHOLOGICAL AND BIOCHEMICAL CHARACTERIZATION OF Rhizobium \\ STRAINS COLLECTED FROM WILD AND DOMESTICATED COMMON BEAN
}

\author{
José de J. López-Alcocer ${ }^{1}$, Rogelio Lépiz-Ildefonso²*, Diego R. González-Eguiarte², \\ Ramón Rodríguez-Macias ${ }^{2}$, Eduardo López-Alcocer ${ }^{2}$ y Víctor Olalde-Portugal ${ }^{3}$
}

\begin{abstract}
'Doctorado en Biosistemática, Ecología y Manejo de Recursos Naturales y Agrícolas, ${ }^{2}$ Centro Universitario de Ciencias Biológicas y Agropecuarias, Universidad de Guadalajara. Camino Ing. Ramón Padilla Sánchez No. 2100. Las Agujas, Zapopan, Jalisco. ${ }^{3}$ Centro de Investigación y de Estudios Avanzados del Instituto Politécnico Nacional. Km 9.6 Carr. Irapuato León. 36821, Irapuato, Guanajuato.
\end{abstract}

*Autor para correspondencia (rlepiz@cucbas.udg.mx)

\section{RESUMEN}

La asociación Rhizobium-leguminosa es considerada un proceso de alta eficiencia en fijación biológica del nitrógeno atmosférico (FBN) y puede ser capaz de abastecer hasta $90 \%$ de las necesidades de nitrógeno en dichas plantas. El objetivo de esta investigación fue realizar la descripción morfológica y bioquímica de 20 cepas de Rhizobium, nueve procedentes de raíces de frijol (Phaseolus vulgaris L.) común silvestre y 11 de raíces de frijol domesticado de la región occidental de México. En la caracterización morfológica de las colonias, todas las cepas exhibieron crecimiento rápido (2 a 3 d) y presentaron color blanco y borde liso, 14 mostraron forma convexa y 12 aspecto traslúcido. En la caracterización bioquímica, todos los rizobios se desarrollaron en medios con valores de $\mathrm{pH}$ iguales o mayores de 6.0 y cuatro no crecieron en $\mathrm{pH}$ de 4.0 a 5.5. En tolerancia a cloruro de sodio, todas las cepas crecieron a $1 \%$ de concentración, 13 lo hicieron a $3 \%$ y ninguna entre 5 y $10 \%$. En resistencia a metales pesados todos los rizobios fueron susceptibles a cloruro de mercurio y sulfato de cobre, ocho fueron resistentes a sulfato de magnesio y sólo dos a sulfatos de magnesio y zinc En resistencia a antibióticos, 16 exhibieron resistencia a los 12 productos utilizados. Las 20 cepas estudiadas mostraron variabilidad y en general presentaron crecimiento rápido, tolerancia a valores de $\mathrm{pH}$ ácido, tolerancia a concentraciones moderadas de cloruro de sodio, susceptibilidad a metales pesados, resistencia a antibióticos y corresponden a bacterias del género Rhizobium. No se observaron diferencias entre las cepas provenientes de frijol silvestre y las provenientes de la forma domesticada.

Palabras clave: Phaseolus vulgaris, Rhizobium, caracterización morfológica, caracterización bioquímica.

\section{SUMMARY}

The Rhizobium-legume association is considered a highly efficient process in biological nitrogen fixation (BNF) and may supply up to $90 \%$ of the nitrogen requirements for those plants. This research described the morphological and biochemical characteristics of 20 Rhizobium strains, nine from wild common bean roots and 11 from domesticated bean roots from Western Mexico. Morphological characterization of colonies showed that all strains exhibited rapid growth ( 2 to $3 \mathrm{~d}$ ), white color and smooth border; 14 had a convex shape, and 12 were translucent. Results from the biochemical characterization indicated that all rhizobia developed at $\mathrm{pH}$ values equal to or greater than 6.0 , and four did not grow in $\mathrm{pH}$ from 4.0 to 5.5 . Strains responded differently to concentrations of sodium chloride: all strains grew at $1 \%, 13$ grew at 3 $\%$ and none grew at 5 to $10 \%$. Resistance to heavy metals showed that all rhizobia were susceptible to mercury chloride and copper sulfate, eight were resistant to magnesium sulfate and only two to magnesium sulfate and zinc sulfate. Sixteen strains exhibited resistance to the 12 antibiotic products used. The 20 strains in this study showed variability and generally presented rapid growth, tolerance to acid $\mathrm{pH}$ values, tolerance to moderate concentrations of sodium chloride, susceptibility to heavy metals, resistance to antibiotics and correspond to bacteria of the genus Rhizobium. No differences were observed between strains from wild beans and those from domesticated ones.

Key words: Phaseolus vulgaris, Rhizobium, morphological characterization, biochemical characterization.

\section{INTRODUCCIÓN}

Las bacterias del género Rhizobium tienen la capacidad de inducir en las raíces de las leguminosas la formación de estructuras especializadas Ilamadas nódulos, dentro de las cuales el $\mathrm{N}_{2}$ atmosférico, que es muy estable y relativamente inerte, se reduce a iones amonio $\left(\mathrm{NH}_{4}^{+}\right)$fácilmente asimilables por la mayoría de las especies vegetales (Marquina et al., 2011).

La asociación Rhizobium-leguminosa se considera un proceso de alta eficiencia en fijación biológica del nitrógeno atmosférico (FBN); se estima que la fijación de nitrógeno varía de 24 hasta $584 \mathrm{~kg} \mathrm{ha}^{-1}$ y tiene la capacidad de abastecer hasta $90 \%$ de las necesidades de la planta (Ángeles-Núñez y Cruz-Acosta, 2015); además, la FBN contribuye a reducir el uso de fertilizantes nitrogenados, a remediar los problemas de contaminación del suelo y agua, y a disminuir los costos de producción, lo cual representa una ventaja económica, a la par de una buena práctica ambiental en la producción agrícola (Granda et al., 2014; Yadegari y Rahmani, 2010).

Las especies del género Rhizobium son bacilos móviles Gram-negativos con pared celular, flagelados (1 a 6 flagelos), aerobios que miden 0.5-0.9 × 1.2-3.0 $\mu \mathrm{m}$, y pueden ser peritricales o subpolares. Las colonias generalmente 
son blancas o color beige, circulares, convexas, semitraslúcidas u opacas y mucilaginosas, que miden de 2 a $4 \mathrm{~mm}$ de diámetro de los 3 a los 5 d de incubación en extracto de levadura manitol agar rojo congo (ELMARC) (Abd-Alla et al., 2012).

El estudio de la taxonomía de los rizobios se basa en varios aspectos como caracterización morfológica, bioquímica y fisiológica, entre otros, y no se pueden adelantar otros como eficiencia en la fijación de $\mathrm{N}$, si no se ha demostrado que pertenecen al género Rhizobium e identificado sus características fenotípicas (Granda et al., 2014; Villanueva y Quintana, 2012).

Los estudios de caracterización morfológica, bioquímica o molecular en el género Rhizobium se han utilizado con diferentes y múltiples propósitos. Destacan los trabajos para evaluar su efectividad potencial (capacidad de fijar $\mathrm{N}_{2}$ ) y capacidad infectiva (inducción de nodulación) (Ángeles-Núñez y Cruz-Acosta, 2015; Mendoza y Bonilla, 2014); diversidad genética (Aserse et al., 2012; Granda et al., 2009); resistencia a metales (Abd-Alla et al., 2012); taxonomía (Gauri et al., 2011; Wei et al., 2003); y para uso como bioinóculo (Cuadrado et al., 2009; Pérez et al., 2008).

Los estudios de caracterización morfológica de cepas de Rhizobium aisladas de nódulos de frijol común (Phaseolus vulgaris $L$.) han incluido las variables de velocidad de crecimiento, color, apariencia, tipo de borde y elevación de las colonias. Sosa et al. (2004) señalan que las colonias de Rhizobium se observan blancas o ligeramente rosadas, forman colonias circulares, son de forma convexa, semitraslúcidas y de crecimiento rápido. Sostienen que las bacterias del género Bradyrhizobium forman colonias circulares, secas, raramente traslúcidas, blancas, convexas, con tendencia a ser granulosas y de crecimiento lento, de 5 a 7 d. En Ecuador, Granda et al. (2013) investigaron la identidad de 45 aislados, 24 de los cuales presentaron características morfológicas pertenecientes al género Rhizobium, crecimiento rápido (2 a 3 d), coloración blanca o rosada, bordes lisos y ondulados y figura elevada y plana.

Por su parte Villanueva y Quintana (2012) estudiaron variables morfológicas en 10 aislados de rizobios nativos de Perú; señalan que seis de las muestras presentaron características referentes al género Rhizobium: consistencia gelatinosa, color blanquecino y velocidad de crecimiento entre 3 y 5 d. Keneni et al. (2010) hicieron una caracterización morfológica y bioquímica de cinco aislados nativos de Rhizobium procedentes de frijol común del norte de Etiopía y dos cepas de alta eficiencia en fijación. Los aislados nativos fueron tolerantes a concentraciones de $5 \%$ de sal, mostraron mayor resistencia a antibióticos y mayor eficiencia en fijación biológica de nitrógeno. Los autores indicaron que los aislados no presentaron crecimiento a niveles de $\mathrm{pH}$ menores de 4.

Se han efectuado caracterizaciones morfológicas y bioquímicas de aislados de Rhizobium procedentes de otras especies de leguminosas. El estudio realizado por Carpio et al. (2014 Com. Pers.) ${ }^{1}$ con aislados de rizobios procedentes de cinco especies de leguminosas: chícharo (Pisum sativum L.), lupinos (Lupinus spp.), frijol común (Phaseolus vulgaris L.), haba (Vicia faba) y Vicia sp., encontraron que las bacterias asociadas a frijol, chícharo, haba y Vicia sp. presentaron características correspondientes al género Rhizobuim, mientras que los rizobios aislados en lupinos mostraron rasgos del género Bradyrhizobium. Por otra parte, Berrada et al. (2012) realizaron estudios de caracterización de 110 cepas de Rhizobium procedentes de leguminosas de grano y forrajeras. Encontraron que 81 de los aislados estudiados fueron de crecimiento rápido, 44 presentaron tolerancia a concentración de $2 \%$ de $\mathrm{NaCl}$ e indicaron que la mayoría presentaron tolerancia a pH de 4 a 8.8, resistencia a antibióticos y a metales pesados (zinc, cobre, níquel y mercurio).

Con base en la diversidad genética de Phaseolus vulgaris silvestre en la región occidente de México (Lépiz-Ildefonso y Ramírez-Delgadillo, 2010) y la presencia de bacterias de Rhizobium asociadas a diferentes formas de frijol común, el objetivo del presente estudio fue la caracterización morfológica y bioquímica de una colecta de 20 cepas de Rhizobium aisladas de nódulos de raíces de plantas de frijol silvestre y domesticado.

\section{MATERIALES Y MÉTODOS}

\section{Colecta de nódulos y purificación de las cepas de Rhizobium}

La colecta de nódulos se realizó en seis localidades de los estados de Jalisco y Michoacán en los meses de septiembre y octubre de 2012 (Cuadro 1). Se extrajeron raíces de cuatro plantas en etapa de floración por sitio de colecta, a una profundidad de $20 \mathrm{~cm}$; las raíces de cada planta se colocaron en una bolsa de plástico, la muestra se identificó y se registraron los datos de tipo de suelo, ubicación geográfica y tipo de vegetación. En el laboratorio se lavaron con agua; de cada raíz se seleccionaron de cinco a seis nódulos grandes, color rosado, superficie rugosa y con estrías claras y blancas; se lavaron en solución jabonosa y

'Carpio M. J., B. M. Paucar y S. P. Alvarado 0. (2013) Caracterización morfológica de rizobios asociados a cultivos de arveja (Pisum sativum L.), chocho (Lupinus mutabilis S.), fréjol (Phaseolus vulgaris L.), haba (Vicia faba L.), y vicia (Vicia atropurpurea) en suelos del Ecuador. In: Memorias de la II Conferencia Iberoamericana de Interacciones Beneficiosas Microorganismo-Planta-Ambiente. Sociedad Española de Fijación de Nitrógeno. Sevilla, España. pp:1-2. 
se desinfectaron con alcohol etílico al $70 \%$ por 1 min; se enjuagaron con agua destilada estéril, se les transfirió a una solución de hipoclorito de sodio al $2 \%$ durante 3 min y finalmente se lavaron cinco veces con agua destilada estéril para retirar el exceso de cloro (Rodríguez, 1993; Somasegaran y Hoben, 1994).

Los nódulos lavados y desinfectados de cada una de las raíces, se depositaron en un tubo de ensayo con $5 \mathrm{~mL}$ de agua destilada y se maceraron con una varilla de vidrio. De cada tubo se tomó una muestra de la suspensión y se sembró en estrías en cajas de Petri en medio extracto de levadura manitol agar rojo congo (ELMARC). Las cajas se incubaron a $28^{\circ} \mathrm{C}$ por un periodo de $5 \mathrm{~d}$, para el crecimiento de las colonias de bacterias (Ángeles-Núñez y CruzAcosta, 2015; Vincent, 1970)

Para la purificación de los aislados, de cada caja se seleccionaron aquellas colonias blancas o ligeramente rosadas, con poca o nula absorción de rojo congo, característica de las bacterias de Rhizobium (Pérez et al., 2008). Las colonias seleccionadas se resembraron cinco veces en cajas de Petri siguiendo el mismo procedimiento. Para su mantenimiento y conservación, los aislados se sembraron en medio extracto de levadura manitol agar (ELMA) en tubos de ensayo a $4{ }^{\circ} \mathrm{C}$ (Rodríguez, 1993).

\section{Caracterización de los aislados}

\section{Caracterización morfológica}

Se incluyeron seis variables a nivel de colonia: velocidad de crecimiento (d), forma (convexa, cupular), color (blanco, rosado), aspecto (traslúcido, opaco), borde (liso, rugoso) y textura (gomosa, acuosa) (Cuadro 1). Para la caracterización se siguió la metodología descrita por el CIAT (1988). Los aislados se sembraron en cajas de Petri con medio ELMA, en dos cajas por aislado. Las cajas se incubaron a $28^{\circ} \mathrm{C}$ por un periodo de $5 \mathrm{~d}$ hasta observar crecimiento de las bacterias y entonces se procedió a evaluar el comportamiento de las colonias.

\section{Caracterización bioquímica}

Se realizaron cuatro pruebas: tolerancia a pH, tolerancia a cloruro de sodio $(\mathrm{NaCl})$, resistencia a metales pesados y

Cuadro 1. Caracterización morfológica de 20 cepas de Rhizobium colectadas en plantas de frijol común (Phaseolus vulgaris L.) silvestre y domesticado en los estados de Jalisco y Michoacán, México.

\begin{tabular}{|c|c|c|c|c|c|c|c|c|}
\hline \multirow{2}{*}{ Código de aislado } & \multirow{2}{*}{ Sitio de origen } & \multirow{2}{*}{$\begin{array}{c}\text { Forma de } \\
\text { frijol }\end{array}$} & \multirow{2}{*}{$\begin{array}{c}\text { Velocidad } \\
\text { crecimiento (d) }\end{array}$} & \multicolumn{5}{|c|}{ Características } \\
\hline & & & & Forma & Color & Aspecto & Borde & Textura \\
\hline Rhizojal VC1 & Nevado de Colima & $S$ & 2 & Convexa & Blanco & Traslúcida & Liso & Gomosa \\
\hline Rhizojal VC2 & Nevado de Colima & S & 2 & Convexa & Blanco & Traslúcida & Liso & Gomosa \\
\hline Rhizojal VC3 & Nevado de Colima & S & 2 & Convexa & Blanco & Traslúcida & Liso & Gomosa \\
\hline Rhizojal BH1 & Huentitán & S & 2 & Convexa & Blanco & Traslúcida & Liso & Gomosa \\
\hline Rhizojal BH2 & Huentitán & S & 2 & Convexa & Blanco & Traslúcida & Liso & Gomosa \\
\hline Rhizojal BH3 & Huentitán & S & 2 & Convexa & Blanco & Traslúcida & Liso & Gomosa \\
\hline Rhizojal SA1 & San Andrés & S & 3 & Cupular & Blanco & Traslúcida & Liso & Acuosa \\
\hline Rhizojal SA2 & San Andrés & $S$ & 3 & Cupular & Blanco & Traslúcida & Liso & Acuosa \\
\hline Rhizojal SA3 & San Andrés & S & 3 & Cupular & Blanco & Traslúcida & Liso & Acuosa \\
\hline Rhizojal ZMA1 & Zapopan & $\mathrm{D}$ & 3 & Convexa & Blanco & Opaca & Liso & Gomosa \\
\hline Rhizojal ZMA2 & Zapopan & D & 3 & Convexa & Blanco & Opaca & Liso & Gomosa \\
\hline Rhizojal ZGZ2 & Zapopan & $\mathrm{D}$ & 3 & Convexa & Blanco & Opaca & Liso & Gomosa \\
\hline Rhizojal ZGZ3 & Zapopan & D & 3 & Convexa & Blanco & Opaca & Liso & Gomosa \\
\hline Rhizojal ZCB3 & Zapopan & $\mathrm{D}$ & 2 & Cupular & Blanco & Traslúcida & Liso & Acuosa \\
\hline Rhizojal ZAT1 & Zapopan & $\mathrm{D}$ & 2 & Cupular & Blanco & Traslúcida & Liso & Acuosa \\
\hline Rhizojal ZAT2 & Zapopan & D & 2 & Cupular & Blanco & Traslúcida & Liso & Acuosa \\
\hline Rhizojal TP1 & Tizapán & $\mathrm{D}$ & 3 & Convexa & Blanco & Opaca & Liso & Gomosa \\
\hline Rhizojal TP3 & Tizapán & $\mathrm{D}$ & 3 & Convexa & Blanco & Opaca & Liso & Gomosa \\
\hline Rhizomich CR1 & Cojumatlán & $\mathrm{D}$ & 2 & Convexa & Blanco & Opaca & Liso & Gomosa \\
\hline Rhizomich CR2 & Cojumatlán & $\mathrm{D}$ & 2 & Convexa & Blanco & Opaca & Liso & Gomosa \\
\hline
\end{tabular}

Todos los sitios pertenecen al estado de Jalisco, excepto Cojumatlán, Michoacán. S: frijol silvestre; D: frijol domesticado. 
resistencia a antibióticos (Cuadros 2, 3 y 4). En todos los casos y antes de cada prueba, los aislados se cultivaron en tubos de ensayo que contenían medio líquido extracto de levadura manitol (CELM) (Rodríguez, 1993), se colocaron en agitación a 200 rpm hasta alcanzar un crecimiento mínimo de $10^{6} \mathrm{UFC} \mathrm{mL}^{-1}$, concentración medida por comparación con la escala de McFarland (1907). En todos los casos se utilizó $1.0 \mathrm{~mL}$ de la suspensión, dilución que se distribuyó uniformemente con una varilla de vidrio en las cajas de Petri con medio ELMA. Igualmente, después de la inoculación de las bacterias en las cajas con el tratamiento respectivo, se incubaron a $28^{\circ} \mathrm{C}$ por $5 \mathrm{~d}$ antes de registrar el resultado de la evaluación correspondiente. Se utilizaron tres cajas de Petri por tratamiento. La respuesta de los aislados de Rhizobium a las cuatro pruebas bioquímicas, se registró como positiva (+) cuando hubo crecimiento y negativa (-) cuando no lo hubo.

Para la evaluación por tolerancia a $\mathrm{pH}$, se incluyeron nueve valores del factor: 4.5, 5.0, 5.5, 6.0, 6.5, 7.0, 7.5, 8.0 y 8.5. Los aislados se sembraron en cajas de Petri en medio ELMA, con el pH ajustado a los diferentes valores, con hidróxido de sodio $(\mathrm{NaOH})$ y ácido clorhídrico $(\mathrm{HCl}) 1 \mathrm{~N}$. La tolerancia a cloruro de sodio ( $\mathrm{NaCl}$ ) se evaluó a concentraciones de 1, 2, 3, 5 y $10 \%$.

La prueba de resistencia a metales incluyó cuatro compuestos: sulfato de zinc $\left(\mathrm{ZnSO}_{4} \cdot 7 \mathrm{H}_{2} \mathrm{O}\right)$, sulfato de magnesio $\left(\mathrm{MgSO}_{4} \cdot 7 \mathrm{H}_{2} \mathrm{O}\right)$, cloruro de mercurio $\left(\mathrm{HgCl}_{2}\right)$ y sulfato de cobre $\left(\mathrm{CuSO}_{4} \cdot 5 \mathrm{H}_{2} \mathrm{O}\right)$ en tres concentraciones cada uno: 10 , 50 y $100\left(\mu \mathrm{g} \mathrm{mL}^{-1}\right)$. Una vez realizada la siembra de los aislados en cajas de Petri con medio ELMA, se colocaron los discos de papel filtro humedecido con las diferentes concentraciones de los metales. Los aislados se consideraron resistentes cuando ocurrió crecimiento (+) y sensibles cuando no lo hubo (-).

La evaluación por resistencia a antibióticos incluyó 12 productos contenidos en sensidiscos Gram negativo II (BIO-RAD, modelo 71080580, México), cuyas concentraciones en $\mu \mathrm{g}$ fueron: trimetroprim-sulfametoxazol $(25 \mu \mathrm{g})$, amikacina $(30 \mu \mathrm{g})$, ampicilina $(10 \mu \mathrm{g})$, levofloxacina $(5 \mu \mathrm{g})$, cefalotina $(30 \mu \mathrm{g})$, cefotaxima $(30 \mu \mathrm{g})$, ceftriaxona $(30 \mu \mathrm{g})$, cloranfenicol $(30 \mu \mathrm{g})$, gentamicina $(10 \mu \mathrm{g})$, netilmicina (30 $\mu \mathrm{g})$, cepefime $(30 \mu \mathrm{g})$ y nitrofurantoína $(30 \mu \mathrm{g})$. Como en las anteriores evaluaciones, las cajas de Petri se incubaron por $5 \mathrm{~d}$ antes de proceder a la evaluación.

\section{Análisis estadístico}

La inspección y análisis de los datos de la caracterización morfológica, donde cada rasgo presenta dos opciones posibles en cada factor, se hizo directamente en la matriz de resultados. Con los datos de la caracterización bioquímica obtenidos en los 38 tratamientos producto de las combinaciones de cuatro factores (tolerancia a $\mathrm{pH}$, tolerancia a $\mathrm{NaCl}$, resistencia a metales pesados y resistencia a antibióticos) y los niveles probados, se realizó un análisis de agrupamiento de los 20 aislados mediante el coeficiente de correlación. Para el análisis, la respuesta positiva (+) que significa crecimiento del aislado, se calificó como 1, y la respuesta negativa (-) que significa ausencia de crecimiento, como 0.

\section{RESULTADOS Y DISCUSIÓN}

\section{Caracterización morfológica}

Los resultados de la caracterización morfológica a nivel de colonia se muestran en el Cuadro 1. Las cepas mostraron diferencias en velocidad de crecimiento, forma, aspecto y textura, no así en color (todas blancas) ni borde (todas lisas). En velocidad de crecimiento, todas las cepas mostraron crecimiento rápido, entre 2 y $3 \mathrm{~d}$. Once de ellas completaron su desarrollo en $2 \mathrm{~d}$, incluidas seis provenientes de frijoles silvestres. En cuanto a la forma de las colonias, 14 cepas mostraron forma convexa y seis forma cupular. En aspecto, 12 cepas presentaron apariencia traslúcida y ocho opaca; fue notable que todos los aislados de la forma silvestre de frijol, mostraran aspecto traslúcido. En textura de colonias, 14 de los 20 aislados presentaron textura gomosa. Seis de las nueve cepas de la forma silvestre mostraron esta textura.

Los resultados de caracterización morfológica son similares a los reportados en la literatura (Granda et al., 2013; Keneni et al., 2010; Villanueva y Quintana, 2012) donde se consigna que las colonias de aislados de Rhizobium son de crecimiento rápido (2 a 3 d), blancas o ligeramente rosadas, forman colonias circulares, convexas y de aspecto traslúcido.

En vista de que todos los aislados presentaron color blanco y borde liso, que mostraron crecimiento rápido (1 a 3 d), aspecto traslúcido (60 \%) y textura gomosa (70 \%), además de tener en cuenta lo asentado en la literatura, se puede afirmar que los 20 aislados pertenecen a bacterias del género Rhizobium, y que las cepas procedentes del frijol silvestre y del domesticado son similares.

\section{Caracterización bioquímica}

\section{Tolerancia a pH}

Con excepción de cuatro cepas que no crecieron en $\mathrm{pH}$ de 4.5 a 5.5, 16 mostraron crecimiento en todos los niveles evaluados (Cuadro 2). Estos resultados muestran que las cepas estudiadas presentan tolerancia a $\mathrm{pH}$ ácido 
con valores hasta de 4.5. Resultados similares reportaron Cuadrado et al. (2009) y Marquina et al. (2011) al evaluar aislados de Rhizobium procedentes de frijol cómun; afirman que los aislados de crecimiento rápido pueden desarrollarse a partir de $\mathrm{pH}$ de 4.0.

\section{Tolerancia a cloruro de sodio}

Todas las cepas crecieron a $1 \%$ de concentración, 15 de las 20 a $2 \%, 13$ a $3 \%$ y solo Rhizojal VC2 a 5 \% de concentración (Cuadro 2). Los resultados son similares a lo reportado en la literatura, donde se señala que el crecimiento bacteriano decrece al aumentar la cantidad de sal en el medio (Berrada et al., 2012; Cuadrado et al., 2009; Patil et al., 2014).

\section{Resistencia a metales pesados}

Todas las cepas fueron suseptibles a las concentraciones evaluadas de cloruro de mercurio y sulfato de cobre (Cuadro 3). De las 20 cepas, trece (siete de variedades domesticadas y seis de frijol silvestre) mostraron resistencia a sulfato de magnesio y dos de éstas (Rhizojal VC2 de frijol silvestre y Rhizojal ZGZ3 de frijol domesticado) a sulfato de zinc. Abd-Alla et al. (2012) consignaron resultados similares en los que hicieron referencia a tolerancia a
Zn y susceptibilidad a Hg. En cambio, Berrada et al. (2012) reportaron que 110 aislados de diferentes leguminosas presentaron alta resistencia a diferentes concentraciones de $\mathrm{Zn}(10,20,40)$, Cu $(10,50,100)$ y Hg $(20,25,100) \mu \mathrm{g}$ $\mathrm{mL}^{-1}$. En la presente investigación, ningún aislado exhibió resistencia a cloruro de mercurio, ni a sulfato de cobre. Las diferencias podrían explicarse por las distintas concentraciones utilizadas entre los estudios y por las diferencias genéticas entre las cepas estudiadas.

\section{Resistencia a antibióticos}

Esta prueba mostró a 16 cepas como resistentes a la mayoría de los antibióticos utilizados; Rhizojal TP1 y Rhizomich CR2 procedentes de los alrededores del Lago de Chapala, fueron susceptibles a los 12 antibióticos ensayados (Cuadro 4). Keneni et al. (2010) evaluaron la resistencia de siete cepas de Rhizobium a seis antibióticos; todos mostraron resistencia a cloranfenicol y penicilina y dos mostraron resistencia a cloranfenicol, penicilina, estreptomicina y tetraciclina; además, afirman que los aislados nativos presentaron mayor resistencia a los antibióticos, que los introducidos. También Hernández et al. (2012), quienes estudiaron 12 cepas y seis antibióticos, reportaron que todas las cepas crecieron en presencia de tetraciclina, cloranfenicol, ampicilina y ácido nalidíxico.

Cuadro 2. Tolerancia de 20 cepas de Rhizobium a nueve valores de pH y cinco concentraciones de cloruro de sodio.

\begin{tabular}{|c|c|c|c|c|c|c|c|c|c|c|c|c|c|c|c|c|c|c|c|c|c|}
\hline \multirow[b]{2}{*}{ Factores } & & \multicolumn{20}{|c|}{ Cepas } \\
\hline & $\frac{\mathscr{O}}{\frac{0}{0}} \frac{0}{>}$ & 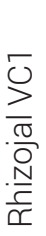 & 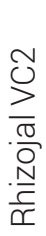 & 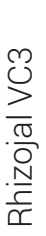 &  & $\begin{array}{l}\frac{N}{D} \\
\frac{T}{\sigma} \\
\frac{D}{O} \\
\frac{N}{\Phi}\end{array}$ & $\begin{array}{l}\frac{m}{\frac{1}{m}} \\
\frac{\bar{\sigma}}{O} \\
\frac{N}{\frac{N}{a}}\end{array}$ & $\begin{array}{l}\frac{\pi}{\sigma} \\
\frac{\pi}{\sigma} \\
\frac{N}{\sigma} \\
\frac{N}{\square}\end{array}$ & 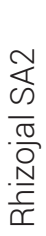 & 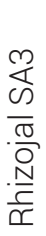 & 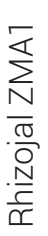 & 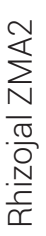 & $\begin{array}{l}N \\
N \\
N \\
N \\
\frac{\sigma}{\sigma} \\
\frac{N}{N} \\
\frac{N}{\widetilde{N}}\end{array}$ & 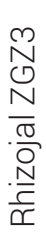 &  & $\begin{array}{l}\text { E } \\
\frac{\pi}{N} \\
\frac{\sigma}{\sigma} \\
\frac{N}{\sigma} \\
\frac{N}{\sim}\end{array}$ & $\begin{array}{l}\text { V } \\
\frac{N}{N} \\
\frac{\sigma}{O} \\
\frac{N}{C} \\
\frac{N}{\simeq}\end{array}$ &  & 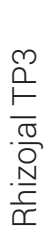 & 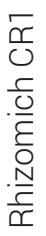 &  \\
\hline \multirow{11}{*}{$\mathrm{pH}$} & 4.5 & + & + & + & + & + & + & + & + & + & - & - & + & + & - & + & + & - & - & + & - \\
\hline & 5.0 & + & + & + & + & + & + & + & + & + & - & - & + & + & + & + & + & - & - & + & + \\
\hline & 5.5 & + & + & + & + & + & + & + & + & + & - & - & + & + & + & + & + & - & - & + & + \\
\hline & 6.0 & + & + & + & + & + & + & + & + & + & + & + & + & + & + & + & + & + & + & + & + \\
\hline & 6.5 & + & + & + & + & + & + & + & + & + & + & + & + & + & + & + & + & + & + & + & + \\
\hline & 7.0 & + & + & + & + & + & + & + & + & + & + & + & + & + & + & + & + & + & + & + & + \\
\hline & 7.5 & + & + & + & + & + & + & + & + & + & + & + & + & + & + & + & + & + & + & + & + \\
\hline & 8.0 & + & + & + & + & + & + & + & + & + & + & + & + & + & + & + & + & + & + & + & + \\
\hline & 8.5 & + & + & + & + & + & + & + & + & + & + & + & + & + & + & + & + & + & + & + & + \\
\hline & 1 & + & + & + & + & + & + & + & + & + & + & + & + & + & + & + & + & + & + & + & + \\
\hline & 2 & + & + & + & + & + & + & + & + & + & - & - & + & + & + & + & - & + & + & - & - \\
\hline \multirow[t]{3}{*}{ NaCl (\%) } & 3 & + & + & + & + & + & + & + & + & + & - & - & + & + & + & + & - & - & - & - & - \\
\hline & 5 & - & + & - & - & - & - & - & - & - & - & - & - & - & - & - & - & - & - & - & - \\
\hline & 10 & - & - & - & - & - & - & - & - & - & - & - & - & - & - & - & - & - & - & - & - \\
\hline
\end{tabular}

+: resistente (hubo crecimiento); -: sensible (no hubo crecimiento). 
Cuadro 3. Evaluación de 20 cepas de Rhizobium por su resistencia a cuatro metales pesados.

\begin{tabular}{|c|c|c|c|c|c|c|c|c|c|c|c|c|c|c|c|c|c|c|c|c|c|}
\hline \multirow[b]{2}{*}{ Productos } & \multirow[b]{2}{*}{$\frac{\mathscr{D}}{\frac{0}{0}}$} & \multicolumn{20}{|c|}{ Cepas } \\
\hline & & $\begin{array}{l}\bar{\nu} \\
\frac{\pi}{\pi} \\
\frac{\pi}{2} \\
\frac{N}{\pi} \\
\frac{\pi}{\alpha}\end{array}$ & 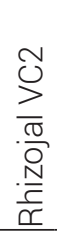 & 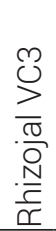 & 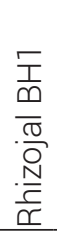 & $\begin{array}{l}\frac{N}{1} \\
\frac{0}{0} \\
\frac{0}{D} \\
\frac{N}{N} \\
\frac{\pi}{\alpha}\end{array}$ & $\begin{array}{l}\frac{m}{I} \\
\frac{m}{0} \\
\frac{\pi}{D} \\
\frac{N}{N} \\
\frac{N}{x}\end{array}$ & $\begin{array}{l}\frac{i}{\Delta} \\
\frac{\omega}{\pi} \\
\frac{\pi}{2} \\
\frac{N}{\frac{N}{x}} \\
\frac{\pi}{x}\end{array}$ & $\begin{array}{l}\frac{N}{c} \\
\frac{\omega}{\sigma} \\
\frac{\pi}{2} \\
\frac{N}{\pi} \\
\frac{N}{\alpha}\end{array}$ & 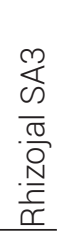 & 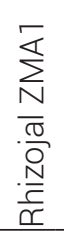 & 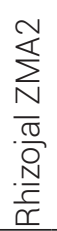 & 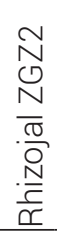 & $\begin{array}{l}N \\
N \\
N \\
N \\
N \\
\frac{N}{N} \\
\frac{N}{N} \\
\frac{N}{\simeq}\end{array}$ & $\begin{array}{l}m \\
0 \\
0 \\
N \\
\frac{\sigma}{D} \\
\frac{N}{N} \\
\frac{N}{\widetilde{C}}\end{array}$ &  & 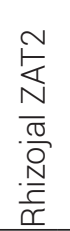 & $\begin{array}{l}\bar{a} \\
\frac{1}{n} \\
\frac{\pi}{2} \\
\frac{N}{\frac{N}{c}} \\
\frac{\pi}{x}\end{array}$ & 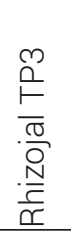 & $\begin{array}{l}\bar{r} \\
0 \\
\frac{c}{0} \\
\frac{0}{E} \\
0 \\
0 \\
\frac{N}{c} \\
\frac{N}{\alpha}\end{array}$ & 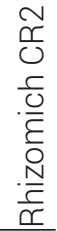 \\
\hline \multirow{3}{*}{$\begin{array}{l}\text { Sulfato de zinc } \\
\left(\mu \mathrm{g} \mathrm{mL}^{-1}\right)\end{array}$} & 10 & - & - & - & + & + & + & - & + & + & - & - & - & + & - & + & - & - & + & + & $\overline{-}$ \\
\hline & 50 & - & + & - & - & - & - & - & - & - & - & - & - & + & - & - & - & - & - & - & - \\
\hline & 100 & - & + & - & - & - & - & - & - & - & - & - & - & + & - & - & - & - & - & - & - \\
\hline \multirow{3}{*}{$\begin{array}{l}\text { Sulfato de } \\
\text { magnesio }(\mu \mathrm{g} \\
\left.\mathrm{mL}^{-1}\right)\end{array}$} & 10 & - & + & - & + & + & + & - & + & + & - & - & + & + & - & + & - & + & + & + & - \\
\hline & 50 & - & + & - & + & + & + & - & + & + & - & - & + & + & - & + & + & + & + & + & - \\
\hline & 100 & - & + & - & + & + & + & - & - & - & - & - & + & - & - & - & + & + & - & - & - \\
\hline \multirow{3}{*}{$\begin{array}{l}\text { Sulfato de cobre } \\
\left(\mu \mathrm{gL}^{-1}\right)\end{array}$} & 10 & - & + & - & - & - & - & - & - & - & - & - & - & - & - & - & - & - & - & - & - \\
\hline & 50 & - & - & - & - & - & - & - & - & - & - & - & - & - & - & - & - & - & - & - & - \\
\hline & 100 & - & - & - & - & - & - & - & - & - & - & - & - & - & - & - & - & - & - & - & - \\
\hline \multirow{3}{*}{ 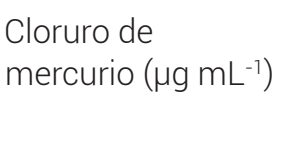 } & 10 & - & - & - & - & - & - & - & - & - & - & - & - & - & - & - & - & - & - & - & - \\
\hline & 50 & - & - & - & - & - & - & - & - & - & - & - & - & - & - & - & - & - & - & - & - \\
\hline & 100 & - & - & - & - & - & - & - & - & - & - & - & - & - & - & - & - & - & - & - & - \\
\hline
\end{tabular}

Cuadro 4. Evaluación de 20 cepas de Rhizobium para resistencia a 12 antibióticos.

\begin{tabular}{|c|c|c|c|c|c|c|c|c|c|c|c|c|c|c|c|c|c|c|c|c|}
\hline \multirow[b]{2}{*}{ Productos } & \multirow[b]{2}{*}{$\begin{array}{l}\bar{U} \\
\bar{\alpha} \\
\frac{\bar{\sigma}}{\Omega} \\
\frac{N}{\pi} \\
\frac{\pi}{\alpha}\end{array}$} & \multicolumn{19}{|c|}{ Cepas } \\
\hline & & $\begin{array}{l}\mathcal{N} \\
\mathcal{D} \\
\frac{\pi}{2} \\
\frac{N}{C} \\
\frac{N}{\sim}\end{array}$ & 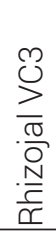 & 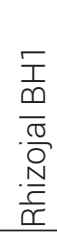 & 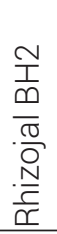 & 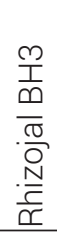 & $\begin{array}{l}\frac{\pi}{a} \\
\frac{1}{0} \\
\frac{\pi}{N} \\
\frac{N}{c} \\
\frac{N}{\alpha}\end{array}$ & 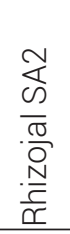 & 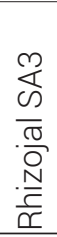 & 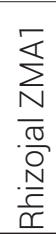 & 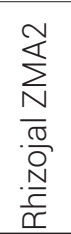 & 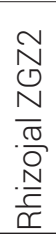 & $\begin{array}{l}N \\
N \\
N \\
N \\
\frac{N}{N} \\
\frac{N}{N} \\
\frac{N}{\mathbb{N}} \\
\frac{1}{\sim}\end{array}$ & 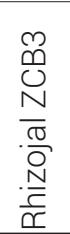 &  & 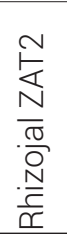 & 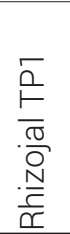 & 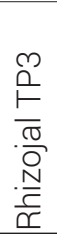 & 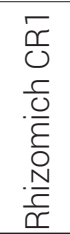 & 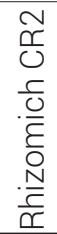 \\
\hline SXT & + & - & + & + & + & + & + & + & + & + & - & + & + & + & + & + & - & - & - & - \\
\hline CTX & + & + & + & + & + & + & + & + & + & + & + & + & + & + & + & + & - & - & + & - \\
\hline CF & + & + & + & + & + & + & + & + & - & + & + & + & + & + & + & + & - & + & + & - \\
\hline FEP & - & + & - & + & - & + & + & + & + & + & + & + & + & + & + & + & - & - & - & - \\
\hline LEV & + & + & + & + & - & - & + & - & + & - & + & + & + & + & - & + & - & - & - & - \\
\hline NF & + & - & + & + & + & + & + & + & - & + & + & + & + & + & + & + & - & + & - & - \\
\hline CRO & + & + & + & + & + & + & + & + & - & + & + & + & + & + & + & + & - & - & + & - \\
\hline AM & + & + & + & + & - & - & + & + & - & + & + & + & + & + & + & + & - & - & - & - \\
\hline NET & + & + & + & + & + & + & + & + & + & + & + & + & + & + & - & + & - & + & + & - \\
\hline$C L$ & + & + & + & + & + & + & + & + & + & + & + & + & + & + & + & + & - & - & - & - \\
\hline AK & + & - & + & + & + & + & + & + & - & + & + & + & + & + & - & + & - & - & - & - \\
\hline GE & + & + & + & + & + & + & + & + & + & + & + & + & + & + & + & + & - & + & + & - \\
\hline
\end{tabular}


Los resultados de la caracterización bioquímica que incluyó tolerancia a pH y a cloruro de sodio, resistencia a metales y resistencia a antibióticos, indican que las cepas estudiadas pertenecen al género Rhizobium.

\section{Análisis de agrupamiento}

El análisis de agrupamiento de las 20 cepas mediante coeficiente de correlación practicado con las 38 variables correspondientes a caracterización bioquímica, dio como resultado la formación de dos grupos principales, $A$ y $B, y$ dentro del grupo A, dos subgrupos A1 y A2 (Figura 1). Dentro de A integrado por 15 cepas, el subgrupo A1 con siete cepas, lo conformaron tres provenientes de frijol silvestre y cuatro de la forma domesticada; la mayoría de las cepas de este subgrupo mostró crecimiento en pH de 4.5, resistencia a metales y resistencia a los antibióticos ensayados. Hubo similitud de resultados entre las cepas Rhizojal VC1 y Rhizojal VC3, y Rhizojal MA1 y Rhizojal MA2, lo que podría indicar que se trata de la misma cepa en cada par, porque fueron colectadas y aisladas de plantas diferentes, pero en el mismo sitio.
Las ocho cepas del subgrupo A2 (cinco procedentes de frijol silvestre), mostraron crecimiento a $\mathrm{pH}$ de 4.5 , a $3 \%$ de $\mathrm{NaCl}$, a las concentraciones de 10 y $50 \mathrm{\mu g} \mathrm{mL}^{-1}$ de sulfato de magnesio y a $1 \mu \mathrm{g} \mathrm{mL}^{-1}$ de sulfato de zinc, excepto Rhizojal ZGZ2. En este grupo destacan Rhizojal BH1, Rhizojal ZGZ2 y Rhizojal ZGZ3 por su resistencia a los 12 antibióticos probados. El alto coeficiente de correlación entre Rhizojal BH2 y Rhizojal BH3 indica que son cepas muy similares.

El grupo B constituido por cuatro cepas aisladas de frijol domesticado procedentes del área del Lago de Chapala, se distinguió por ser susceptible a pH ácido (4.5), a concentraciones bajas de sal (3 \%) y a los antibióticos; además, mostraron similitud de resultados en cinco variables de caracterización morfológica: forma, color, aspecto, borde y textura (Cuadro 1). En el caso de Rhizojal VC2 colectado en raíces de frijol silvestre en el volcán Nevado de Colima, no mostró cercanía con ninguna otra cepa. Creció en todos los niveles de $\mathrm{pH}$ probados, fue el único que se desarrolló a la concentración de $5 \%$ de $\mathrm{NaCl}$ y el único con resistencia a las concentraciones evaluadas de sulfato de zinc y sulfato de

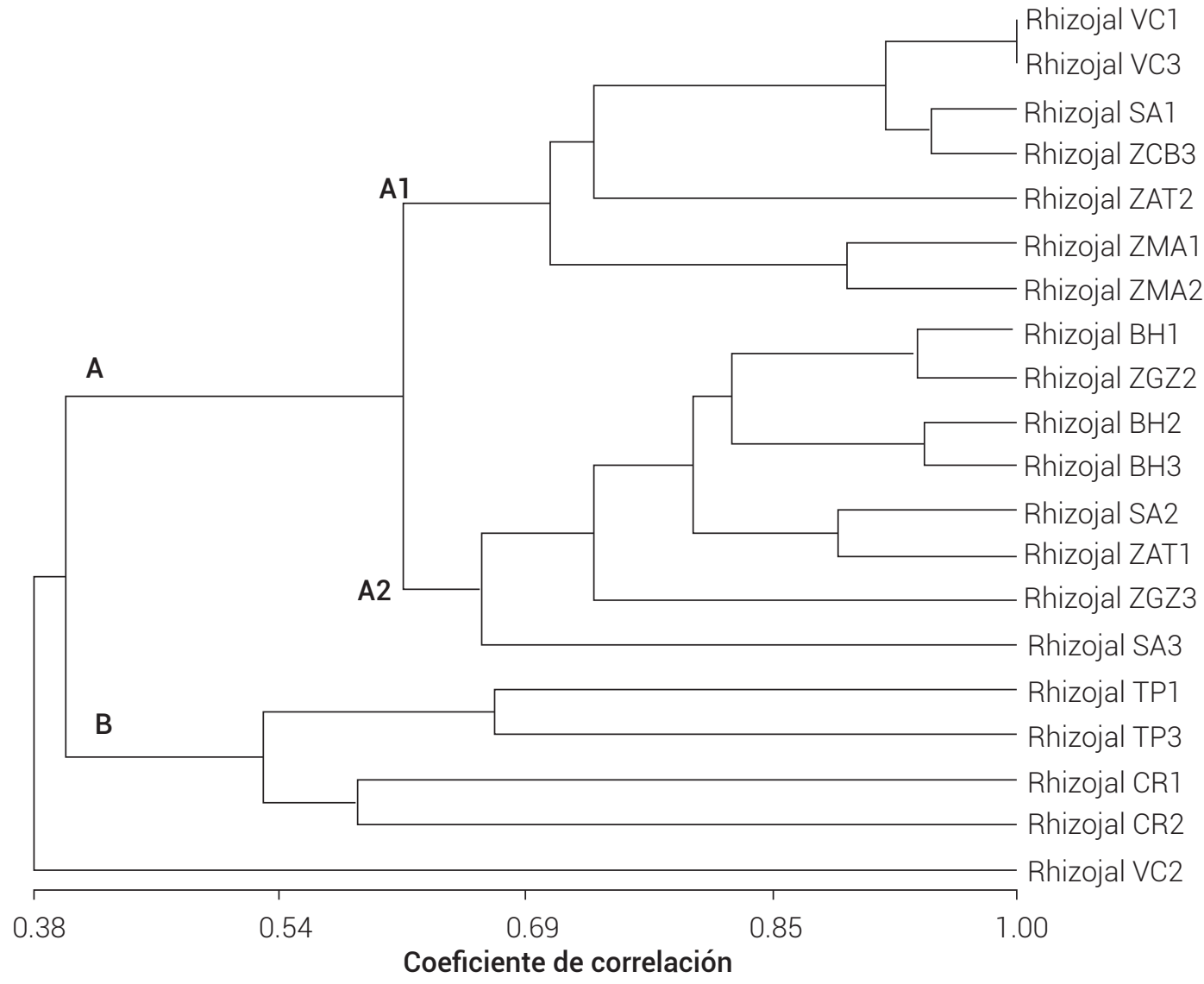

Figura 1. Dendrograma elaborado con 38 variables resultado de la caracterización bioquímica de 20 cepas de Rhizobium mediante coeficiente de correlación. 
magnesio. Se requieren estudios adicionales para verificar si se trata de una especie diferente.

La caracterización morfológica y bioquímica de las cepas estudiadas, evidencian la variabilidad genética de Rhizobium en el occidente de México y alto potencial para seleccionar cepas de Rhizobium de crecimiento rápido, resistentes a factores adversos como acidez del suelo y a niveles moderados de salinidad y con resistencia a antibióticos. Esta afirmación se basa en lo expresado tanto por Hernández et al. (2012), como por Abera et al. (2015), quienes señalan que los factores de supervivencia, persistencia y competitividad confieren a los rizobios ventajas adaptativas para sobrevivir y competir con otras bacterias del suelo, lo que favorece la colonización de las raíces de las leguminosas y la fijación de nitrógeno atmosférico.

\section{CONCLUSIONES}

Los estudios de caracterización practicados a 20 cepas de bacterias, 11 procedentes de nódulos de frijol cultivado y nueve de frijoles silvestres de la región occidente de México, evidenciaron que se trata de bacterias del género Rhizobium. Las cepas exhibieron diferencias morfológicas y bioquímicas, y mostraron variabilidad fenotípica. Las cepas estudiadas presentaron diferencias marcadas de crecimiento en valores distintos de $\mathrm{pH}$, en concentraciones de cloruro de sodio, en reacción a metales pesados y en respuesta a los antibióticos utilizados. Las cepas mostraron de manera general crecimiento rápido, tolerancia a valores de $\mathrm{pH}$ ácido, tolerancia a concentraciones moderadas de cloruro de sodio, susceptibilidad a metales pesados, y resistencia a antibióticos. La caracterización y estudios de agrupamiento de las 20 cepas de Rhizobium no mostraron diferencias morfológicas y bioquímicas entre cepas de poblaciones de frijol silvestre y de variedades domesticadas, con excepción de una cepa procedente de frijol silvestre del Nevado de Colima.

\section{BIBLIOGRAFÍA}

Abd-Alla M. H., F. M. Morsy, A-W. E. El-Enany and T. Ohyama (2012) Isolation and characterization of a heavy-metal-resistant isolate of Rhizobium leguminosarum bv. viciae potentially applicable for biosorption of $\mathrm{Cd}^{2+}$ and $\mathrm{CO}^{2+}$. International Biodeterioration and Biodegradation 67:48-55.

Abera T., E. Semu, T. Debele, D. Wegary and H. Kim (2015) Determination soil Rhizobium populations, intrinsic antibiotic resistance, nodulation and seed yield of faba bean and soybean in Western Ethiopia. World Journal of Agricultural Sciences 11:311-324.

Ángeles-Núñez J. G. y T. Cruz-Acosta (2015) Aislamiento, caracterización molecular y evaluación de cepas fijadoras de nitrógeno en la promoción del crecimiento de frijol. Revista Mexicana de Ciencias Agrícolas 6:929-942.

Aserse A. A., L.A. Räsänen, F. Assefa, A. Hailemariam and K. Lindström (2012) Phylogeny and genetic diversity of native rhizobia nodulating common bean (Phaseolus vulgaris L.) in Ethiopia. Sistematic and Applied Microbiology 35:120-131.

Berrada H., I. Nouioui, M. Iraqui H., N. El Ghachtouli, M. Gtari and K. Fikri B.
(2012) Phenotypic and genotypic characterizations of rhizobia isolated from root nodules of multiple legume species native of Fez, Morocco. African Journal of Microbiology Research 6:5314-5324.

CIAT, Centro Internacional de Agricultura Tropical (1988) Simbiosis leguminosa-rizobio: Manual de Métodos de Evaluación, Selección y Manejo Agronómico. Centro Internacional de Agricultura Tropical. Cali, Colombia. 194 p.

Cuadrado B., G. Rubio y W. Santos (2009) Caracterización de cepas de Rhizobium y Bradyrhizobium (con habilidad de nodulación) seleccionados de los cultivos de fríjol caupi (Vigna unguiculata) como potenciales bioinóculos. Revista Colombiana de Ciencias Químico Farmacéuticas 38:78-104.

Gauri, A. K. Singh, R. P. Batt, S. Pant, M. K. Bedi and A. Naglot (2011) Characterization of Rhizobium isolated from root nodules of Trifolium alexandrinum. Journal of Agricultural Technology 7:1705-1723.

Granda M. K. I., A. Colás S., R. Cupull S., Y. Gutiérrez S. y R. Torres G. (2009) Caracterización e identificación genética de aislados de Rhizobium en el cultivo del fréjol común (Phaseolus vulgaris L.). Centro Agrícola 36:5-14.

Granda M. K., M. Ochoa T., V. Ruilova V., F. Guamán D. y R. Torres G. (2014) Evaluación de cepas nativas de Rhizobium sobre parámetros fenotípicos en frijol común (Phaseolus vulgaris L.). Centro de Biotecnología 3:25-37.

Granda M. K., H. Paccha C., C. Campoverde S. y R. Torres G. (2013) Variabilidad de aislados diazotróficos simbióticos en diferentes condiciones agroecológicas del sur del Ecuador. Centro de Biotecnología 2:6-15

Hernández F. I., M. C. Nápoles G., G. Pérez H., P. R. Rosales J., R. Baños G. y J. F. Ramírez P. (2012) Caracterización fenotípica de aislados de rizobios procedentes de la leguminosa forrajera Canavalia ensiformis. Cultivos Tropicales 33:21-28.

Keneni A., F. Assefa and P. C. Prabu (2010) Characterization of acid and salt tolerant Rhizobial strains isolated from faba bean fields of Wollo, Northern Ethiopia. Journal of Agricultural Science and Technology 12:365-376

Lépiz-Ildefonso R. y R. Ramírez-Delgadillo (2010) Los Parientes Silvestres del Frijol Común en el Occidente de México. Orgánica Editores. Universidad de Guadalajara SINAREFI, Guadalajara, México. 64 p.

Marquina M. E., N. E. González y Y. Castro (2011) Caracterización fenotípica y genotípica de doce rizobios aislados de diversas regiones geográficas de Venezuela. Revista de Biología Tropical 59:1017-1036.

McFarland J. (1907) The nephelometer: an instrument for estimating the number of bacteria in suspensions used for calculating the opsonic index and for vaccines. Journal American Medical Association 49:1176-1178.

Mendoza L. J. A y R. R. Bonilla B. (2014) Infectividad y efectividad de rizobios aislados de suelos de la Costa Caribe colombiana en Vigna unguiculata. Revista Colombiana de Biotecnología XVI:84-89.

Patil S. M., D. B. Patil, M. S. Patil, P. V. Gaikwad, S. B. Bhamburdekar and P. J. Patil (2014) Isolation, characterization and salt tolerance activity of Rhizobium sp. from root nodules of some legumes. International Journal of Current Microbiology and Applied Sciences 3:1005-1008

Pérez G., G. Gómez, M. C. Nápoles y B. Morales (2008) Aislamiento y caracterización de cepas de rizobios aisladas de diferentes leguminosas en la región de Cascajal, Villa Clara. Pastos y Forrajes 31:151-159.

Rodríguez M. M. N. (1993) Asociación Rhizobium-leguminosa In: Manual de Agromicrobiología. R. Ferrera C., M. C. A. González C. y M. N. Rodríguez M. (eds.). Editorial Trillas. México. pp:11-51

Somasegaran P. and H. J. Hoben (1994) Handbook for Rhizobia: Methods in Legume-Rhizobium Technology. NifTAL Project. University of Hawaii Springer. New York. Hawaii, USA. 450 p.

Sosa A., A. Elías, O. A. García y M. Sarmiento (2004) Aislamiento y caracterización fenotípica parcial de cepas de rizobios que nodulan leguminosas rastreras. Revista Cubana de Ciencia Agrícola 38:197-201.

Villanueva T. E. E. y A. Quintana D. (2012) Aislamiento y selección de bacterias nativas de rizobios fijadores de nitrógeno, a partir de nódulos radiculares de Phaseolus vulgaris. REBIOL Revista Científica de la Facultad de Ciencias Biológicas 32:24-30. 
Vincent J. M. (1970) A Manual for the Practical Study of Root-nodule Bacteria. IBP Handbook No. 15. International Biological Programme. Blackwell Scientific, Oxford-Edinburgh.164 p.

Wei G. H., Z. Y. Tan, M. E. Zhu, E. T. Wang, S. Z. Han and W. X. Chen (2003) Characterization of rhizobia isolated from legume species within the genera Astragalus and Lespedeza grown in the Loess Plateau of China and description of Rhizobium loessense sp. nov.
International Journal of Systematic and Evolutionary Microbiology 53:1575-1583.

Yadegari M. and H. A. Rahmani (2010) Evaluation of bean (Phaseolus vulgaris) seeds' inoculation with Rhizobium phaseoli and plant growth promoting Rhizobacteria (PGPR) on yield and yield components. African Journal of Agricultural Research 5:792799 